\title{
AVALIAÇÃO DAS PROPRIEDADES QUÍMICAS EM SOLO DE CERRADO SOB REFLORESTAMENTO CILIAR
}

\author{
Alexandre Marques da Silva ${ }^{1}$, Daniela Sílvia de Oliveira Canuto ${ }^{2}$, Mario Luiz Teixeira de Moraes $^{3}$, \\ Salatiér Buzetti ${ }^{4}$ \\ ${ }^{1}$ Geógrafo, M.Sc., Depto. de Fitotecnia, Tecnologia de Alimentos e Sócio Economia, UNESP, Ilha Solteira, São Paulo, Brasil - \\ amsilva@agr.feis.unesp.br \\ ${ }^{2}$ Bióloga, Dra ., Depto. de Fitotecnia, Tecnologia de Alimentos e Sócio Economia, UNESP, Ilha Solteira, São Paulo, Brasil - \\ dsocanuto@aluno.feis.unesp.br \\ ${ }^{3}$ Eng. Agrônomo, Depto. de Fitotecnia, Tecnologia de Alimentos e Sócio Economia, UNESP, Ilha Solteira, São Paulo, Brasil - \\ teixeira@agr.feis.unesp.br \\ ${ }^{4}$ Eng. Agrônomo, Depto. de Fitossanidade, Engenharia Rural e Solos, UNESP, Ilha Solteira, São Paulo, Brasil -sbuzetti@ agr.feis.unesp.br
}

Recebido para publicação: 16/06/2009 - Aceito para publicação: 21/11/2011

\begin{abstract}
O rápido crescimento da agropecuária, promovido pelas iniciativas públicas, favoreceu um desmatamento descontrolado de nossas florestas, de modo que hoje se faz necessário o reflorestamento de áreas de preservação permanente para a conservação de nossos recursos naturais, sendo a recuperação dessas áreas obrigatória pelas leis públicas. Em uma área de 2,13 ha de reflorestamento ciliar no bioma Cerrado, foram avaliados os atributos químicos do solo após vinte anos de plantio. Os atributos analisados foram: $\mathrm{P}$, matéria orgânica $(\mathrm{MO}), \mathrm{pH}, \mathrm{K}, \mathrm{Ca}, \mathrm{Mg}, \mathrm{H}+\mathrm{Al}, \mathrm{Al}$ e $\mathrm{S}$, em 13 módulos, com 3 repetições, em duas profundidades (0-20 e 20-40 cm), com delineamento em blocos casualizados em esquema hierárquico. O reflorestamento com espécies arbóreas à margem do rio Paraná em Selvíria (MS) contribui para que os atributos químicos do solo, sob o reflorestamento ciliar, sejam semelhantes aos de um solo sem perturbação antrópica nessa região, verificando-se que o plantio das espécies arbóreas promove deposição de material orgânico, o que é fundamental para a ciclagem de nutrientes, mantendo-se, dessa forma, as propriedades químicas desse solo em boas condições para o estabelecimento da mata ciliar.

Palavras-chave: Reflorestamento ciliar; cerrado; mata ciliar; propriedades químicas do solo.
\end{abstract}

Resumo

\begin{abstract}
Evaluation of chemical properties of savanna soil under riparian reforesting. The rapid growth of agriculture, promoted by public government initiatives, favored an out of control deforestation of our forests; today, reforestation of permanent preservation areas are necessary for the conservation of our natural resources, and recovery of such areas are required by public laws. In an area of 5.26 acres of riparian reforestation in the savanna soil chemical properties were evaluated after twenty years of planting. It was analyzed the following variables: $(\mathrm{P}$, organic matter $(\mathrm{MO}), \mathrm{pH}, \mathrm{K}, \mathrm{Ca}, \mathrm{Mg}, \mathrm{H}+\mathrm{Al}, \mathrm{Al}$ and $S)$ in 13 modules, with three replicas in two depths $(0-20$ and $20-40 \mathrm{~cm})$ in a randomized block design in hierarchical scheme. Reforestation with tree species on the edge of Parana river in Selvíria (MS) contributes to chemical soil attributes, under riparian reforestation, as similar as a soil without human disturbance in this region; it is also possible to verify that forestry plantation promotes deposition of organic material which is essential for nutrient cycling, which keep the chemical properties of such soil in good condition for the establishment of riparian vegetation. Keywords: Riparian reforestation, savanna, riparian forest, soil chemical properties.
\end{abstract}

\section{INTRODUÇÃO}

$\mathrm{Na}$ história da humanidade, a exploração excessiva dos recursos naturais, com a substituição gradativa da vegetação original por áreas de cultivos ou pastagens, acabou por criar enormes desertos, tornando o solo mais pobre e incapaz de suprir as necessidades de seus habitantes. O problema agrava-se em muitas regiões onde ocorrem instabilidade climática - principalmente secas prolongadas - e crescimento acelerado da população. 
Entre as principais consequências do desmatamento, podem-se citar: o esgotamento dos estoques de madeiras, a destruição acelerada do solo, a desertificação gradativa, com consequente diminuição da produtividade, a ocorrência de inundações e o aumento do efeito estufa. Sabe-se que a floresta é uma associação de organismos que vivem em equilíbrio, altamente vulnerável às ameaças internas e externas. Qualquer dano pode quebrar a integridade desse ciclo harmônico e provocar uma destruição em cadeia.

Atividades como a exploração de madeira, a agropecuária, a expansão urbana, as queimadas e o manejo inadequado do solo, a mineração e até o turismo e o lazer, sem critérios apropriados, podem prejudicar a floresta. Com a construção de usinas hidrelétricas há o represamento das águas e o avanço delas sobre o ambiente ripário, contribuindo para a perda da floresta ali existente.

O Cerrado stricto sensu é o bioma que vem sofrendo as maiores taxas de desmatamento, devido ao rápido processo de expansão de fronteiras agrícolas do país, atraindo grande parte da agroindústria nacional para essas áreas. Entre as formações vegetais do cerrado, o ambiente fluvial ou ripário caracteriza-se por associar-se aos cursos d'água com elevada riqueza, diversidade genética e pelo seu papel na proteção dos recursos hídricos, edáficos, fauna silvestre e aquática.

Embora sejam áreas de preservação permanente, protegidas por legislação estadual e federal (BARBOSA et al., 1992), as matas ciliares, também chamadas de mata de galeria ou ripária (JOLY, 1970; AB'SABER, 1971), vêm sofrendo alterações significativas desde 1850 (BAITELLO et al., 1983/85), encontrando-se, em geral, extremamente degradadas (MARTINS, 1989). De acordo com Kageyama e Dias (1982), as intensas e desordenadas devastações podem levar ao desaparecimento de diversas espécies florestais de importância ecológica e comercial em várias regiões, com sério comprometimento de seu potencial genético. Sendo assim, a sua restauração, utilizando-se espécies vegetais adequadas a essa finalidade, é uma necessidade urgente e imprescindível para a conservação dos recursos hídricos de bacias hidrográficas alteradas (SALVADOR, 1989).

Se um ecossistema vai ser recuperado, é porque perdeu suas características fundamentais; logo, o primeiro passo, tanto na definição dos objetivos quanto no desenho de modelos de plantio até a avaliação dos resultados, é a seleção de informações de referência quanto à sua estrutura e função, que no caso de matas ciliares são: redução de perda de solo decorrente de processos erosivos e de solapamentos das margens dos rios causados pela sua ausência; fornecimento de locais de refúgios e fontes de alimentação para as faunas silvestre e aquática; promoção do repovoamento faunístico quando recompostas artificialmente; proteção dos cursos d'água de impactos decorrentes de transportes de defensivos, corretivos e fertilizantes; melhoria da qualidade e aumento da quantidade de água para consumo humano e uso agrí́cola (ENGEL; PARROTTA, 2003; CRESTANA et al., 2004).

Sob florestas ciliares ocorre uma significativa variação de solos, cujos reflexos aparecem nos diversos tipos de formações florestais, variando desde as de terrenos mais encharcados (floresta paludosa ou mata de brejo) até os mais secos, onde as florestas apresentam características florísticas e fisionomias distintas, de acordo com o gradiente de umidade e influência fluvial no solo. Além desses aspectos, há também variação em porte, estrutura e composição florística dessas formações. Variações em função do solo são detectadas, entretanto faltam informações de correlações específicas entre os diversos solos e as diversas variações das formações florestais ocorrentes nessas situações, principalmente as relações específicas (JACOMINE, 2004). Curcio et al. (2007) concluíram que a composição e a estrutura florística, assim como a ocupação da paisagem fluvial pelas fitotipias floresta e front hidrófilo pioneiro, estão diretamente relacionadas à altura e à forma das feições geomórficas, assim como às características dos tipos de solos, com ênfase ao seu regime hídrico.

Com a cobertura do solo pela vegetação ciliar, este se torna mais poroso e menos denso, propiciando um melhor desenvolvimento das espécies arbóreas. Portanto, a intervenção do homem em determinados ambientes degradados acelera a sua recuperação. Há também uma diminuição na temperatura do solo, favorecendo a vida microbiana, vida esta importantíssima para o ecossistema do solo.

Para recuperação da cobertura florestal às margens dos rios em regiões de domínio de cerrado, recomenda-se o plantio de espécies adaptadas às condições desfavoráveis de fertilidade do solo dessas regiões (DURIGAN; SILVEIRA, 1999). A deposição de serapilheira por espécie caducifólia em matas ciliares favorece a ciclagem de nutrientes, aumentando a produção da biomassa, propiciando com isso que o ciclo se feche. Esse aumento de matéria orgânica melhora as condições físicas do solo.

A proposta do presente trabalho tem como objetivo avaliar as propriedades químicas de um solo sob vegetação de cerrado em reflorestamento ciliar após vinte anos de plantio, e, como hipótese, que, se o 
reflorestamento com espécies arbóreas ocorrer em área de mata ciliar, então os atributos químicos do solo se mantêm de modo equivalente a uma área sem perturbação antrópica.

\section{MATERIAL E MÉTODOS}

O trabalho foi realizado no reflorestamento ciliar da Fazenda de Ensino, Pesquisa e Extensão (FEPE) da Faculdade de Engenharia de Ilha Solteira, Universidade Estadual Paulista "Júlio de Mesquita Filho" (FEIS/UNESP), localizada no município de Selvíria (MS).

A área da fazenda foi cedida pela Companhia Energética do Estado de São Paulo (CESP) em comodato para a UNESP, em 1977, para que fossem desenvolvidas pesquisas agronômicas. No mesmo ano, iniciou-se o desflorestamento até a margem do rio Paraná, área ciliar que sofreu intensiva mecanização agrícola com o plantio de arroz. Essa atividade só parou com a instalação do reflorestamento, em 1986.

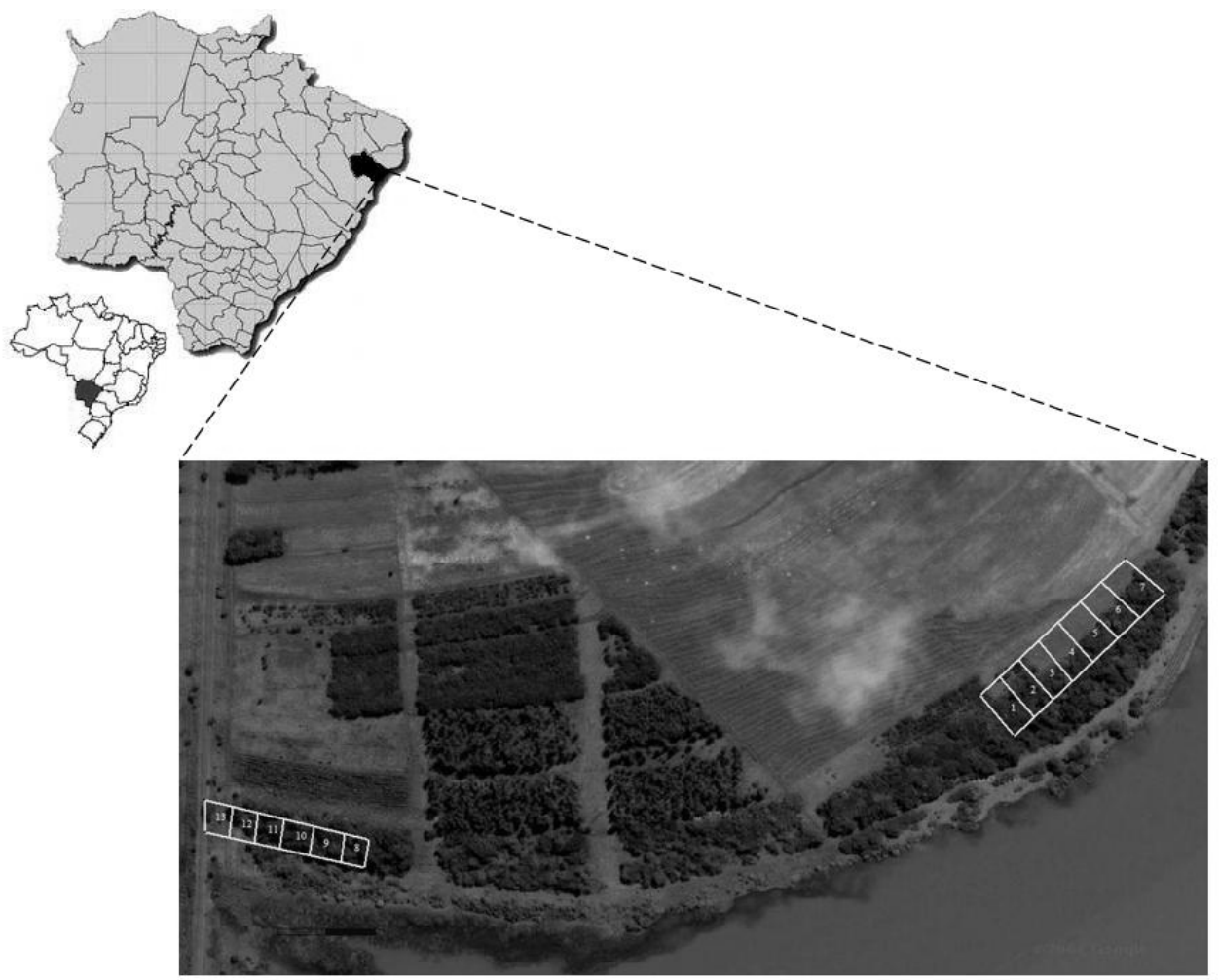

Figura 1. Localização geográfica do reflorestamento ciliar estudado no município de Selvíria (MS).

Figure 1. Geographical location of riparian reforestation in Selvíria (MS).

O clima da região é classificado como Aw, segundo o sistema de Köppen, apresentando chuvas no verão e seca no inverno. A área de estudo, que se encontra no Planalto da Bacia Sedimentar do Paraná, apresenta declives muito suaves, relevo plano e suavemente ondulado (ALVES, 2001), enquanto o solo, pela nomenclatura atual em nível de subordem, é um LATOSSOLO VERMELHO (EMBRAPA, 1999).

O reflorestamento ciliar foi plantado em módulos nos meses de fevereiro e março de 1986, com espaçamento de $2 \times 3 \mathrm{~m}$, com área total dos módulos de 2,13 ha. As mudas para o plantio foram doadas pela CESP, compostas por 14 espécies arbóreas plantadas em 13 módulos (Figura 1).

O experimento possui 11 linhas de plantio. Para montar o modelo estatístico, consideraram-se as nove linhas centrais divididas em três, portanto, a cada três linhas tem-se uma repetição. As linhas I e XI são bordaduras; as linhas II, III e IV, repetição 1; linhas V, VI e VII, repetição 2; e as linhas VIII, IX e X, repetição 3. Isso foi usado em todos os módulos de plantio (Figura 2). 
Tabela1. Croqui da divisão em repetições de um módulo do reflorestamento à margem do reservatório da Usina Hidrelétrica de Ilha Solteira.

Table1. Sketch of division in repetition of a module of reforestation on the edge of Hydroelectrical Plant of Ilha Solteira.

\begin{tabular}{cccccccccccccccc}
\hline Repetições & Linhas & \multicolumn{11}{c}{ Tratamentos (espécies) } \\
\hline Bordadura & I & 14 & 9 & 4 & 3 & 6 & 1 & 5 & 13 & 2 & 12 & 10 & 7 & 8 & 11 \\
& II & 9 & 11 & 8 & 2 & 12 & 7 & 10 & 4 & 1 & 13 & 6 & 14 & 3 & 5 \\
1 & III & 9 & 11 & 7 & 12 & 4 & 3 & 8 & 6 & 10 & 14 & 13 & 5 & 1 & 2 \\
& IV & 7 & 6 & 14 & 8 & 13 & 5 & 10 & 9 & 2 & 11 & 3 & 1 & 4 & 12 \\
& V & 10 & 8 & 7 & 3 & 6 & 4 & 12 & 1 & 2 & 9 & 11 & 14 & 13 & 5 \\
2 & VI & 11 & 8 & 2 & 6 & 9 & 10 & 12 & 7 & 4 & 13 & 3 & 1 & 14 & 5 \\
& VII & 5 & 12 & 11 & 7 & 2 & 6 & 13 & 1 & 8 & 3 & 10 & 4 & 9 & 14 \\
& VIII & 9 & 11 & 1 & 7 & 13 & 5 & 4 & 8 & 14 & 10 & 12 & 6 & 2 & 3 \\
3 & IX & 1 & 14 & 5 & 11 & 7 & 9 & 6 & 4 & 3 & 8 & 12 & 10 & 13 & 2 \\
& X & 9 & 3 & 7 & 14 & 12 & 1 & 2 & 8 & 6 & 13 & 4 & 10 & 5 & 11 \\
Bordadura & XI & 1 & 8 & 6 & 11 & 2 & 12 & 13 & 3 & 4 & 10 & 5 & 14 & 7 & 9 \\
\hline
\end{tabular}

A identificação das espécies utilizadas no reflorestamento ciliar está apresentada na tabela 2 .

Tabela 2. Espécies utilizadas no reflorestamento ciliar à margem do reservatório da Hidrelétrica de Ilha Solteira.

Table 2. Species used in the riparian reforesting on the edge of Hydroelectrical Plant of Ilha Solteira.

\begin{tabular}{llll}
\hline IDT & Nome científico & Nome vulgar & Família/Subfamília \\
\hline 1 & Anadenanthera peregrina var. falcata & Angico-roxo & Mimosaceae \\
2 & Myracrodruon urundeuva & Aroeira & Anacardiaceae \\
3 & Cordia ecalyculata & Café-de-bugre & Boraginaceae \\
4 & Mabea fistulifera & Canudo-de-pito & Euphorbiaceae \\
5 & Terminalia argentea & Capitão-do-campo & Combretaceae \\
6 & Genipa americana & Jenipapo & Rubiacea \\
7 & Inga sp. & Ingá & Mimosaceae \\
8 & Tabebuia sp. & Ipê-amarelo-do-campo & Bignoniaceae \\
9 & Tabebuia heptaphylla & Ipê-rosa & Bignoniaceae \\
10 & Michelia champaca & Magnólia-amarela & Magnoliaceae \\
11 & Eriobotrya japonica & Nespereira & Rosaceae \\
12 & Adenanthera pavonina & Olho-de-dragão & Fabaceae \\
13 & Leucana leucocephala & Leucena & Mimosoideae \\
14 & Melia azedarach & Santa-bárbara & Meliaceae \\
\hline
\end{tabular}

IDT: Identificação utilizada no campo.

As análises químicas do solo foram obtidas a partir de amostras deformadas nas profundidades de 0-20 e 20-40 cm, em cada módulo, e determinadas segundo metodologia descrita em Raij e Quaggio (1983). Determinaram-se fósforo, potássio, cálcio e magnésio pelo método de extração com resina trocadora de íons. A matéria orgânica foi determinada por meio do método colorimétrico. $\mathrm{O}$ hidrogênio mais alumínio, pela solução tampão SMP, e o $\mathrm{pH}$, por solução de $\mathrm{CaCl}_{2} 0,01 \mathrm{M}$.

O delineamento estatístico utilizado foi o de blocos casualizados completos em esquema hierárquico (Tabela 3).

Para a análise estatística das propriedades químicas do solo, foram utilizadas a análise de variância, o teste de F e as comparações das médias pelo teste de Scott e Knott (1974), procedida com o pacote estatístico SISVAR (FERREIRA, 2004).

\section{RESULTADOS E DISCUSSÃO}

A análise de variância indicou que houve diferenças estatísticas para o efeito do desdobramento de módulos dentro de profundidades para matéria orgânica, K, Al, soma de bases e capacidade de troca 
catiônica. Para as profundidades estudadas, não houve diferença estatística apenas para o pH e Al. Para o efeito do desdobramento de repetições dentro de profundidades, houve diferença estatística somente para matéria orgânica (Tabela 4).

Tabela 3. Esquema da análise de variância utilizada na análise das variáveis químicas do solo.

Table 3. Scheme of analysis for variance used in the analysis of soil chemical variables.

\begin{tabular}{lccc}
\hline FV* & GL & QM & F \\
\hline Repetições (R)/P & $(\mathrm{r}-1) \mathrm{p}$ & $\mathrm{Q}_{1}$ & $\mathrm{Q}_{1} / \mathrm{Q}_{4}$ \\
Módulos (M)/P & $(\mathrm{m}-1) \mathrm{p}$ & $\mathrm{Q}_{2}$ & $\mathrm{Q}_{2} / \mathrm{Q}_{4}$ \\
Profundidade (P) & $(\mathrm{p}-1)$ & $\mathrm{Q}_{3}$ & $\mathrm{Q}_{3} / \mathrm{Q}_{4}$ \\
Erro & $\mathrm{p}(\mathrm{r}-1)(\mathrm{m}-1)$ & $\mathrm{Q}_{4}$ & - \\
\hline Total & $\mathrm{rmp}-1$ & $\mathrm{Q}_{5}$ & - \\
\hline
\end{tabular}

* R: repetições; M: módulos; P: profundidade.

Tabela 4. Quadrados médios, coeficientes de variação e médias para as propriedades químicas do solo sob reflorestamento ciliar, aos 20 anos após o plantio.

Table 4. Mean squares, and coefficient of variation for the chemical properties of soil under riparian reforestation, 20 years after planting.

\begin{tabular}{|c|c|c|c|c|c|c|c|}
\hline \multirow{2}{*}{ FV } & \multirow{2}{*}{ GL } & $\mathbf{P}$ & MO & pH & $\mathbf{K}$ & $\mathbf{C a}$ & Mg \\
\hline & & mg.dm ${ }^{-3}$ & g.dm ${ }^{-3}$ & $\mathrm{CaCl}_{2}$ & \multicolumn{3}{|c|}{$\mathrm{mmol}_{\mathrm{c} \cdot \mathrm{dm}^{-3}}$} \\
\hline Repetição (Profundidade) & 4 & $0^{\mathrm{ns}}$ & $10^{*}$ & $0,02^{\mathrm{ns}}$ & $0,01^{\mathrm{ns}}$ & $0^{\mathrm{ns}}$ & ns \\
\hline Módulo (Profundidade) & 24 & $0^{\mathrm{ns}}$ & $16^{* *}$ & $0,04^{\mathrm{ns}}$ & $1,71 * *$ & $0^{\mathrm{ns}}$ & ns \\
\hline Profundidade & 1 & $2 * *$ & $1354 * *$ & $0,00^{\mathrm{ns}}$ & $2,92 * *$ & $10 * *$ & $* *$ \\
\hline Erro & 48 & 0 & 3 & 0,02 & 0,06 & 0 & 0 \\
\hline $\mathrm{CV}(\%)$ & & 18,63 & 8,72 & 3,18 & 16,66 & 17,31 & 19,15 \\
\hline Média & & 2 & 20 & 4,3 & 2,2 & 10 & 5 \\
\hline \multirow{2}{*}{ FV } & \multirow{2}{*}{ GL } & $\mathbf{H}+\mathbf{A l}$ & Al & SB & $\mathbf{T}$ & $\mathbf{V}$ & $\mathbf{S}$ \\
\hline & & \multicolumn{4}{|c|}{$\operatorname{mmol}_{\mathbf{c} .} \cdot \mathbf{d m}^{-3}$} & $\%$ & mg.dm ${ }^{-3}$ \\
\hline Repetição (Profundidade) & 4 & $110^{\mathrm{ns}}$ & $0^{\mathrm{ns}}$ & $0^{\mathrm{ns}}$ & $94^{\mathrm{ns}}$ & $0^{\mathrm{ns}}$ & $0^{\mathrm{ns}}$ \\
\hline Módulo (Profundidade) & 24 & $145^{\mathrm{ns}}$ & $0 *$ & $1 * *$ & $235^{* *}$ & $1^{\mathrm{ns}}$ & $1^{\mathrm{ns}}$ \\
\hline Profundidade & 1 & $1473 * *$ & $0^{\mathrm{ns}}$ & $18 * *$ & $5383 * *$ & $6 * *$ & $19 * *$ \\
\hline Erro & 48 & 84 & 0 & 0 & 70 & 1 & 1 \\
\hline $\mathrm{CV}(\%)$ & & 16,96 & 21,04 & 16,37 & 11,73 & 16,03 & 45,07 \\
\hline Média & & 54 & 5 & 17 & 71 & 24 & 5 \\
\hline
\end{tabular}

FV: fonte de variação; GL: graus de liberdade; P: fósforo; MO: matéria orgânica; K: potássio; Ca: cálcio; Mg: magnésio; H+Al: hidrogênio+alumínio; Al: alumínio; SB: soma de bases; T: capacidade de troca catiônica; V: saturação por bases; S: enxofre.

ns: não significativo; *: significativo a $5 \%$; **: significativo a $1 \%$. Quadrados médios e CV das variáveis $\mathrm{P}, \mathrm{K}, \mathrm{Ca}, \mathrm{Mg}, \mathrm{Al}, \mathrm{SB}, \mathrm{V}$ e S foram transformados em $\sqrt{x+0,5}$.

Com os resultados da tabela 4, observou-se que o solo é naturalmente ácido, assim como já confirmado por Cavalcante (1999), Cavenage et al. (1999), Souza (2000). Mas, de maneira geral, está se mantendo uma deposição de material orgânico, o que garante o suprimento de nutrientes às espécies que ali se encontram, juntamente com os nutrientes que são carreados das áreas agrícolas para o reflorestamento ciliar.

Mesmo apresentando uma média de pH que expressa uma acidez alta, os valores dos macronutrientes são suficientes para suprir as necessidades das espécies que ali se encontram.

São apresentados os valores médios do desdobramento de repetição dentro de profundidade na tabela 5. Houve diferença estatística apenas para matéria orgânica e Al, utilizando o teste de Scott e Knott, nas propriedades químicas do solo.

O teor de matéria orgânica foi maior na repetição 1 na profundidade de $0-20 \mathrm{~cm}$. Isso ocorre, provavelmente, em função de uma maior sobrevivência de plantas nessa repetição, e pode ser também devido ao acúmulo de material orgânico carreado das áreas altas, que fica retido no reflorestamento. 
Tabela 5. Valores médios para as propriedades químicas do solo de profundidade dentro de repetição sob reflorestamento ciliar, aos 20 anos após o plantio.

Table 5. Average values for chemical properties of soil in depth, within repetition under riparian reforestation, 20 years after planting.

\begin{tabular}{|c|c|c|c|c|c|c|c|c|c|c|c|c|c|}
\hline \multirow{2}{*}{ Profundidade* } & \multicolumn{6}{|c|}{ REP } & \multirow{3}{*}{ Profundidade } & \multicolumn{6}{|c|}{ REP } \\
\hline & \multicolumn{2}{|c|}{1} & \multirow{2}{*}{\multicolumn{2}{|c|}{$\frac{2}{\left(\mathrm{mg.dm}^{-3}\right)}$}} & \multicolumn{2}{|c|}{3} & & \multicolumn{2}{|c|}{1} & & & & 3 \\
\hline & & & & & & & & \multicolumn{6}{|c|}{$\mathrm{H}+\mathrm{Al} \overline{\left(\mathrm{mmol}_{\mathrm{c}} \cdot \mathrm{dm}^{-3}\right)}$} \\
\hline 1 & 3 & A & $2^{\circ}$ & A & 2 & A & 1 & 60 & A & 57 & A & 58 & A \\
\hline \multirow[t]{2}{*}{2} & 1 & A & 1 & A & 1 & A & 2 & 54 & A & 48 & A & 47 & A \\
\hline & \multicolumn{6}{|c|}{ MO $\left(\mathrm{g.dm}^{-3}\right)$} & & \multicolumn{6}{|c|}{$\mathrm{Al}\left(\mathrm{mmol}_{\mathrm{c}} \cdot \mathrm{dm}^{-3}\right)$} \\
\hline 1 & 26 & B & 24 & A & 24 & A & 1 & 5 & A & 5 & A & 5 & A \\
\hline \multirow[t]{2}{*}{2} & 16 & A & 17 & $\mathrm{~A}$ & 16 & $\mathrm{~A}$ & 2 & 6 & $\mathrm{~B}$ & 4 & $\mathrm{~A}$ & 5 & $\mathrm{~A}$ \\
\hline & \multicolumn{6}{|c|}{$\mathrm{pH}\left(\mathrm{CaCl}_{2}\right)$} & & \multicolumn{6}{|c|}{ SB $\left(\right.$ mmol $\left._{\mathbf{c}} \cdot \mathrm{dm}^{-3}\right)$} \\
\hline 1 & 4,2 & A & 4,3 & A & 4,3 & A & 1 & 22 & A & 22 & A & 21 & A \\
\hline \multirow[t]{2}{*}{2} & 4,2 & A & 4,3 & A & 4,3 & A & 2 & 12 & A & 15 & A & 13 & A \\
\hline & \multicolumn{6}{|c|}{$K\left(\mathrm{mmol}_{\mathrm{c}} \cdot \mathrm{dm}^{-3}\right)$} & & \multicolumn{6}{|c|}{$\mathrm{T}\left(\mathrm{mmol}_{\mathrm{c}} \cdot \mathrm{dm}^{-3}\right)$} \\
\hline 1 & 2,5 & A & 2,9 & A & 3,0 & A & 1 & 81 & A & 79 & A & 78 & A \\
\hline \multirow[t]{2}{*}{2} & 1,5 & A & 1,6 & A & 1,7 & A & 2 & 67 & A & 63 & A & 60 & A \\
\hline & \multicolumn{6}{|c|}{$\mathrm{Ca}\left(\mathrm{mmol}_{\mathrm{c}} . \mathrm{dm}^{-3}\right)$} & & \multicolumn{6}{|c|}{$\mathrm{V} \%$} \\
\hline 1 & 13 & A & 13 & A & 12 & A & 1 & 27 & A & 27 & A & 26 & A \\
\hline \multirow[t]{2}{*}{2} & 7 & $\mathrm{~A}$ & 8 & $\mathrm{~A}$ & 8 & $\mathrm{~A}$ & 2 & 19 & A & 23 & A & 21 & $\mathrm{~A}$ \\
\hline & \multicolumn{6}{|c|}{$\mathrm{Mg}\left(\mathrm{mmol}_{\mathrm{c}} \cdot \mathrm{dm}^{-3}\right)$} & & \multicolumn{6}{|c|}{$\mathrm{S}\left(\mathrm{mg.dm}^{-3}\right)$} \\
\hline 1 & 7 & A & 7 & A & 6 & A & 1 & 2 & A & $2^{\circ}$ & A & 2 & A \\
\hline 2 & 3 & A & 4 & A & 3 & A & 2 & 6 & A & 7 & A & 9 & A \\
\hline
\end{tabular}

* 1: 0-20 cm; 2: 20-40 cm. P: fósforo; MO: matéria orgânica; K: potássio; Ca: cálcio; Mg: magnésio; H+Al: hidrogênio+alumínio; Al: alumínio; SB: soma de bases; T: capacidade de troca catiônica; V: saturação por bases; S: enxofre. Médias não seguidas por mesma letra diferem entre si pelo Teste de Scott e Knott a 5\% de probabilidade.

A maior concentração de $\mathrm{Al}$ foi verificada na profundidade de $20-40 \mathrm{~cm}$ na repetição 1 . Nesta, a soma de bases não refletiu em redução da acidez desse solo.

Os resultados obtidos para as propriedades químicas do solo, utilizando o teste de Scott e Knott, demonstraram que não houve diferença estatística nas propriedades químicas do solo para $\mathrm{P}, \mathrm{Ca}, \mathrm{H}+\mathrm{Al}$ e S (Tabela 6).

Os maiores teores de matéria orgânica ocorreram com maiores frequências nos módulos 8 ao 13, nas duas profundidades estudadas. Os teores de matéria orgânica apresentaram-se médios, conforme Lopes (1984), e são valores semelhantes ao solo natural dessa região (CAVALCANTE, 1999; CAVENAGE et al., 1999). Observa-se que as espécies estão depositando matéria orgânica suficiente para a manutenção do reflorestamento ciliar e, mesmo não sendo procedentes de área de cerrado, estão tolerando as condições ácidas desse solo.

Os valores de $\mathrm{pH}$ e $\mathrm{Mg}$ só diferiram pelo teste de Scott e Knott na profundidade de 0-20 cm, e o $\mathrm{K}$ diferiu nas duas profundidades estudadas. Assim como ocorreu com os valores de matéria orgânica, os maiores valores desses elementos no solo ocorrem do módulo 8 ao módulo 13. De maneira geral, verificou-se que esses módulos apresentaram suas propriedades químicas de solo melhores do que os demais módulos, visto que se encontram separados por certa distância e em posições diferentes no relevo, o que possibilita receber, com maiores frequências, produtos químicos de áreas adjacentes que, consequentemente, contribuem para afetar as propriedades químicas do solo.

Os valores encontrados para essas propriedades químicas do solo são maiores quando comparados com os valores do solo natural, encontrados por autores como Cavenage et al. (1999), Cavalcante (1999) e Souza (2000). Naturalmente, se os valores de pH são maiores nos módulos de 8 a 13, a acidez é menor e, portanto, os valores de Al nesses módulos são baixos na profundidade de $0-20 \mathrm{~cm}$. Na profundidade de 20-40 cm, os valares médios foram semelhantes entre os módulos estudados.

As condições químicas do solo desse reflorestamento ciliar garantem a disponibilidade de nutrientes às plantas que ali se encontram. Portanto, as espécies plantadas estão bem estabelecidas no local, mesmo que algumas não pertençam ao bioma de cerrado. Esses solos geralmente são ácidos e apenas espécies dessa região suportam tais condições. $\mathrm{O}$ suprimento de $\mathrm{Ca}$ pode estar relacionado à 
grande quantidade de resíduos provenientes da parte aérea e também das calagens que são realizadas nas áreas agrícolas próximas do reflorestamento. Toniato et al. (1998), no estudo de fitossociologia de um remanescente de floresta higrófila em Campinas (SP), encontraram no fragmento estudado valores de $16,00 \mathrm{mmol}_{\mathrm{c}} \cdot \mathrm{dm}^{-3}$ na borda da mata e $11,60 \mathrm{mmol}_{\mathrm{c}} \cdot \mathrm{dm}^{-3} \mathrm{em}$ seu interior. Portanto, o entorno do reflorestamento tem que ser considerado um fator de grande influência na ciclagem de nutrientes e estabelecimento dessas espécies.

Tabela 6. Valores médios do desdobramento de módulos dentro de profundidades para as propriedades químicas do solo sob reflorestamento ciliar, aos 20 anos após o plantio.

Table 6. Average values for unfolding of modules within depths, for chemical properties of soil under riparian reforestation, 20 years after planting.

\begin{tabular}{|c|c|c|c|c|c|c|c|c|c|c|c|c|c|c|c|c|c|c|c|c|c|c|c|}
\hline \multirow{3}{*}{ Módulo } & \multicolumn{3}{|c|}{$\begin{array}{c}P \\
\text { mg.dm } \\
\end{array}$} & \multicolumn{4}{|c|}{$\begin{array}{c}\text { MO } \\
\text { g.dm }^{-3}\end{array}$} & \multicolumn{4}{|c|}{$\begin{array}{c}\mathrm{pH} \\
\mathrm{CaCl}_{2}\end{array}$} & \multicolumn{4}{|c|}{$\mathbf{K}$} & \multicolumn{4}{|c|}{$\begin{array}{c}\mathrm{Ca} \\
\text { mmol }_{\mathrm{c} .} \cdot \mathrm{dm}^{-3}\end{array}$} & \multicolumn{4}{|c|}{$\mathbf{M g}$} \\
\hline & \multicolumn{23}{|c|}{ Profundidades } \\
\hline & 1 & & 2 & \multicolumn{2}{|c|}{1} & \multicolumn{2}{|c|}{2} & \multicolumn{2}{|l|}{1} & \multicolumn{2}{|c|}{2} & \multicolumn{2}{|c|}{1} & \multicolumn{2}{|c|}{2} & \multicolumn{2}{|c|}{1} & \multicolumn{2}{|c|}{2} & \multicolumn{2}{|c|}{1} & \multicolumn{2}{|c|}{2} \\
\hline 1 & $2 \mathrm{~A}$ & 1 & A & 26 & B & 14 & A & 4,2 & A & 4,3 & A & 0,8 & A & 0,0 & A & 13 & A & 9 & A & 8 & B & 3 & A \\
\hline 2 & $2 \mathrm{~A}$ & 1 & A & 22 & A & 14 & A & 4,1 & A & 4,2 & A & 0,1 & A & 0,0 & A & 11 & A & 8 & A & 5 & A & 3 & A \\
\hline 3 & $3 \mathrm{~A}$ & 1 & A & 25 & B & 17 & B & 4,1 & A & 4,3 & A & 0,5 & A & 0,0 & A & 11 & A & 7 & A & 5 & A & 4 & A \\
\hline 4 & $2 \mathrm{~A}$ & 1 & $\mathrm{~A}$ & 22 & A & 14 & A & 4,1 & A & 4,2 & A & 0,3 & A & 0,0 & A & 11 & A & 7 & A & 5 & A & 3 & A \\
\hline 5 & $2 \mathrm{~A}$ & 1 & A & 24 & A & 17 & B & 4,2 & A & 4,3 & A & 0,7 & A & 0,0 & A & 12 & A & 7 & A & 6 & A & 3 & A \\
\hline 6 & $2 \mathrm{~A}$ & 1 & A & 23 & A & 15 & A & 4,2 & A & 4,3 & A & 0,9 & A & 0,0 & A & 13 & A & 9 & A & 5 & A & 2 & A \\
\hline 7 & $2 \mathrm{~A}$ & 1 & A & 20 & A & 14 & A & 4,2 & A & 4,3 & A & 0,3 & A & 0,0 & A & 8 & A & 7 & A & 4 & A & 2 & A \\
\hline 8 & $2 \mathrm{~A}$ & 1 & A & 30 & $\mathrm{C}$ & 17 & B & 4,1 & A & 4,2 & A & 2,0 & B & 0,4 & A & 14 & A & 7 & A & 8 & B & 2 & A \\
\hline 9 & $2 \mathrm{~A}$ & 1 & A & 28 & $\mathrm{C}$ & 17 & B & 4,3 & A & 4,3 & A & 4,5 & $\mathrm{C}$ & 2,0 & B & 12 & A & 9 & A & 8 & B & 3 & A \\
\hline 10 & $3 \mathrm{~A}$ & 1 & A & 26 & B & 17 & B & 4,5 & B & 4,3 & A & 6,2 & $\mathrm{D}$ & 3,3 & B & 16 & A & 8 & A & 8 & B & 4 & A \\
\hline 11 & $3 \mathrm{~A}$ & 2 & A & 25 & B & 18 & B & 4,5 & B & 4,4 & A & 8,2 & $\mathrm{D}$ & 6,4 & $\mathrm{C}$ & 16 & A & 8 & A & 8 & B & 4 & A \\
\hline 12 & $5 \mathrm{~A}$ & 2 & A & 25 & B & 18 & B & 4,4 & B & 4,3 & A & 6,5 & $\mathrm{D}$ & 5,4 & $\mathrm{C}$ & 15 & A & 10 & A & 10 & B & 4 & A \\
\hline 13 & $3 \mathrm{~A}$ & 2 & A & 23 & A & 17 & B & 4,4 & B & 4,2 & A & 5,1 & $\mathrm{C}$ & 3,0 & B & 10 & A & 6 & A & 10 & B & 3 & $\mathrm{~A}$ \\
\hline \multirow{4}{*}{ Módulos } & \multirow{2}{*}{\multicolumn{3}{|c|}{$\mathrm{H}+\mathrm{Al}$}} & \multicolumn{4}{|c|}{ Al } & & S & B & & & 1 & 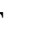 & & & $\mathbf{V}$ & & & & $S$ & & \\
\hline & & & & & & & & c. $\mathrm{dm}^{-}$ & & & & & & & & & $\%$ & & & & mg.c & & \\
\hline & & & & & & & & & & & rof & ad & & & & & & & & & & & \\
\hline & 1 & & 2 & & 1 & & 2 & 1 & 1 & 2 & & 1 & & 2 & & 1 & & 2 & & 1 & & 2 & \\
\hline 1 & 58 & A & 62 & A & 6 & B & 6 A & 22 & B & 11 & A & 80 & B & 73 & B & 27 & B & 18 & A & 3 & A & 13 & A \\
\hline 2 & 67 & A & 45 & A & 8 & B & 6 A & 16 & A & 11 & A & 82 & B & 56 & A & 19 & A & 19 & A & 2 & A & 8 & A \\
\hline 3 & 62 & A & 44 & A & 7 & B 4 & $4 \mathrm{~A}$ & 14 & A & 14 & A & 76 & A & 58 & A & 19 & A & 23 & A & 2 & A & 5 & A \\
\hline 4 & 58 & A & 42 & A & 8 & B 4 & $4 \mathrm{~A}$ & 14 & A & 11 & A & 72 & A & 53 & A & 20 & A & 21 & A & 2 & A & 9 & A \\
\hline 5 & 57 & A & 45 & A & 5 & B 4 & $4 \mathrm{~A}$ & 16 & A & 14 & A & 72 & A & 58 & A & 22 & A & 24 & A & 2 & A & 10 & A \\
\hline 6 & 49 & A & 36 & A & 4 & B & $4 \mathrm{~A}$ & 19 & A & 11 & A & 67 & A & 47 & A & 28 & B & 23 & A & 3 & A & 4 & A \\
\hline 7 & 54 & A & 49 & A & 6 & B & $4 \mathrm{~A}$ & 12 & B & 9 & A & 66 & A & 59 & A & 18 & A & 17 & A & 2 & A & 7 & A \\
\hline 8 & 69 & A & 54 & A & 7 & B 7 & $7 \mathrm{~A}$ & 24 & B & 10 & A & 92 & B & 64 & B & 26 & B & 15 & A & 2 & A & 8 & A \\
\hline 9 & 65 & A & 51 & A & 5 & B & $6 \mathrm{~A}$ & 22 & B & 17 & A & 87 & B & 68 & B & 26 & B & 25 & A & 1 & A & 7 & A \\
\hline 10 & 53 & A & 53 & A & 1 & A 5 & $5 \mathrm{~A}$ & 30 & B & 16 & A & 82 & B & 68 & B & 35 & B & 23 & A & 1 & A & 2 & A \\
\hline 11 & 51 & A & 48 & A & 2 & A & $4 \mathrm{~A}$ & 32 & B & 18 & A & 83 & B & 66 & B & 38 & B & 27 & A & 2 & A & 4 & A \\
\hline 12 & 61 & A & 63 & A & 4 & A 8 & $8 \mathrm{~A}$ & 31 & B & 20 & A & 92 & B & 83 & B & 34 & B & 24 & A & 2 & A & 13 & A \\
\hline 13 & 56 & $\mathrm{~A}$ & 54 & A & 3 & A & $6 \quad \mathrm{~A}$ & 25 & $\mathrm{~B}$ & 12 & $\mathrm{~A}$ & 81 & B & 66 & $\mathrm{~B}$ & 31 & B & 18 & A & 1 & A & 1 & A \\
\hline
\end{tabular}

* 1: 0-20 cm; 2: 20-40 cm. P: fósforo; MO: matéria orgânica; K: potássio; Ca: cálcio; Mg: magnésio; H+Al: hidrogênio+alumínio; Al: alumínio; SB: soma de bases; T: capacidade de troca catiônica; V: saturação por bases; S: enxofre. Médias não seguidas por mesma letra diferem entre si pelo Teste de Scott e Knott a 5\% de probabilidade.

Os valores maiores para as propriedades químicas do solo provavelmente podem estar relacionados à deposição de serapilheira, que vem acontecendo durante esses vinte anos, com a instalação do reflorestamento ciliar. A decomposição e liberação de nutrientes da serapilheira foliar em um trecho da floresta ombrófila densa aluvial em regeneração em Guaraqueçaba (PR) foi crescente ao longo do tempo de decomposição da serapilheira entre os macronutrientes N, P, Ca e Mg (SHEER, 2008). 
Dickow et al. (2009) concluíram que o aumento na liberação de nutrientes através da lixiviação das folhas da serapilheira à medida que a sucessão vegetal evolui esteve relacionado às características morfológicas das folhas e às condições de sítio favoráveis a uma maior decomposição do material vegetal na fase avançada. $\mathrm{O}$ fato de as espécies terem se comportado de maneira diferenciada em relação à ciclagem dos nutrientes é de relevante importância ecológica, pois garante a manutenção de um equilíbrio biogeoquímico no ecossistema.

Os maiores valores dos elementos estudados encontram-se na profundidade de $0-20 \mathrm{~cm}$, com exceção do $\mathrm{S}$, que naturalmente ocorre no subsolo devido à adsorção não específica do elemento (Tabela 7). Esses valores, na primeira profundidade estudada, são um indicativo da ciclagem dos nutrientes pela floresta, que está completamente estabelecida no local de estudo, e, também, devido aos resíduos de produtos agrícolas que são retidos pela matéria seca e se acumulam no solo.

Pissara-Franco (1994) encontrou os maiores valores para as propriedades químicas do solo na camada de 0-20 cm em reflorestamento no reservatório da Hidrelétrica de Ilha Solteira, em área de empréstimo, o que realmente confirma os valores aqui determinados.

Tabela 7. Valores médios para as profundidades estudadas em função das propriedades químicas do solo sob reflorestamento ciliar, aos 20 anos após o plantio.

Table 7. Average values for the focused depths depending on soil chemical properties under riparian reforestation, 20 years after planting.

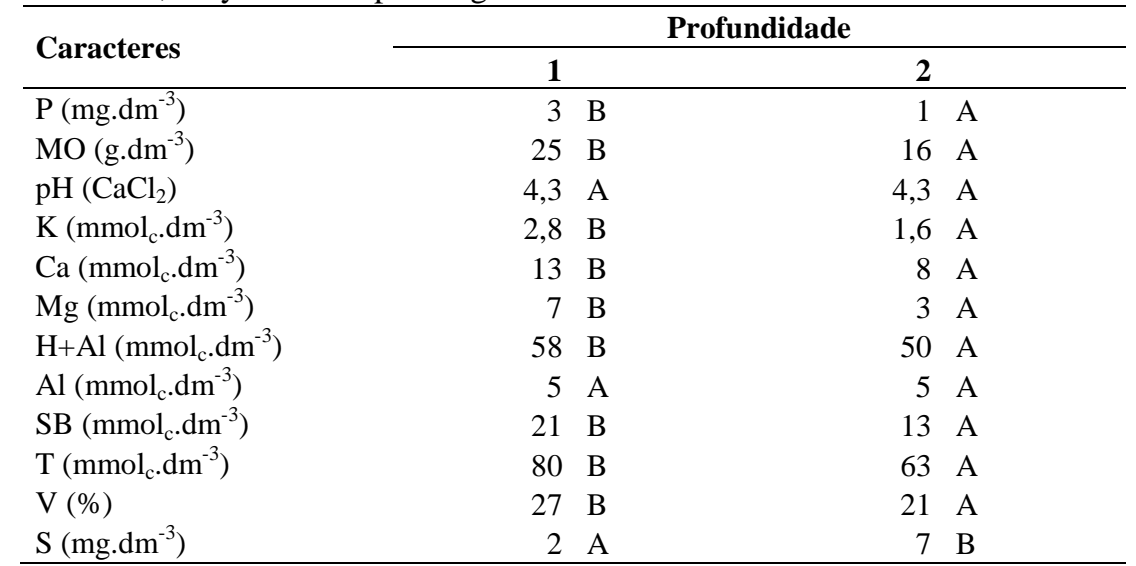

\section{CONCLUSÕES}

- O reflorestamento com espécies arbóreas à margem do rio Paraná em Selvíria (MS) contribui para que os atributos químicos do solo sob o reflorestamento ciliar sejam semelhantes aos de um solo sem perturbação antrópica nessa região.

- Após 20 anos da instalação do reflorestamento ciliar, verifica-se que o plantio das espécies arbóreas promove deposição de material orgânico, o que é fundamental para a ciclagem de nutrientes, mantendo-se, dessa forma, as propriedades químicas desse solo em boas condições para o estabelecimento da mata ciliar.

\section{AGRADECIMENTOS}

À Fundação para o Desenvolvimento da UNESP (FUNDUNESP), pelo auxílio financeiro para o desenvolvimento da pesquisa.

\section{REFERÊNCIAS}

AB'SABER, A. N. Contribuição à geomorfologia da área do cerrado. In: SIMPÓSIO SOBRE O CERRADO, 1971, São Paulo. Anais... São Paulo: S.n., 1971. p. 97 - 103. 
ALVES, M. C. Recuperação do subsolo de um latossolo vermelho usado para terrapleno e fundação da Usina Hidrelétrica de Ilha Solteira, SP. Ilha Solteira, 2001. 83 p. Tese (Livre-Docente) - Faculdade de Engenharia de Ilha Solteira, Universidade Estadual Paulista, Ilha Solteira, 2001.

BAITEllo, J. B.; AGUIAR, O. T.; PASTORE, J. A. Essências florestais da Reserva Estadual da Cantareira - São Paulo - Brasil. Silvicultura em São Paulo, São Paulo, v. 17/19, p. 61 - 84, 1983/85.

BARBOSA, L. M.; ASPERTI, L. M.; BEDINELLI, C.; BARBOSA, J. M.; BELASQUE, E. F.; PIRRÉ, E. Informações básicas para modelos de recuperação de áreas degradadas de matas ciliares. In: CONGRESSO NACIONAL SOBRE ESSÊNCIAS NATIVAS, 4, 1992, São Paulo. Anais... São Paulo: Revista do Instituto Florestal, 1992. p. 640 - 644.

CAVALCANTE, E. G. S. Variabilidade espacial de atributos físicos e químicos de um Latossolo Vermelho-Escuro sob diferentes condições de uso e manejo, em Selvíria (MS). 1999. 199 f. Dissertação (Mestrado) - Faculdade de Engenharia de Ilha Solteira, Universidade Estadual Paulista, Ilha Solteira, 1999.

CAVENAGE, A.; MORAES, M. L. T; ALVES, M. C.; CARVALHO, M. A. C.; FREITAS, M. L. M.; BUZETTI, S. Alterações das propriedades físicas de um Latossolo Vermelho-Escuro sob diferentes culturas. Revista Brasileira de Ciência do Solo, Viçosa, MG, v. 23, n. 4, p. 997 - 1003. 1999.

CRESTANA, M. S. M. (org.); FERRETI, A. R.; TOLEDO FILHO, D. V.; ÁRBOCZ, G. F.; SCHIMIDT, H. A. P.; GUARDIA, J. F. C. Florestas: sistemas de recuperação com essências nativas, produção de mudas e legislações. 2.ed. Campinas: CATI, 2004. 216 p.

CURCIO, G. R.; GALVÃO, F.; BONNET, A.; BARDDAL, M. L.; DEDECEK, R. A. A floresta fluvial em dois compartimentos do rio Iguaçu, Paraná, Brasil. Floresta, Curitiba, PR, v. 37, n. 2, p. 125 - 147, 2007.

DICKOW, K. M.; MARQUES, R.; PINTO, C. B. Lixiviação de nutrientes da serapilheira recémdepositada em sucessão ecológica na Floresta Atlântica, litoral do Paraná. Floresta, Curitiba, PR, v. 39, n. 1 , p. $145-156,2009$.

DURIGAN, G.; SILVEIRA, E. R. Recomposição da mata ciliar em domínio de cerrado, Assis, SP. Scientia Forestalis, Piracicaba, n. 56, p. 135 - 144, 1999.

EMPRESA BRASILEIRA DE PESQUISA AGROPECUÁRIA (EMBRAPA). Sistema brasileiro de classificação de solos. Rio de Janeiro: EMBRAPA/CNPSO, 1999. 412 p.

ENGEL, V. L.; PARROTTA, J. A. Definindo a restauração ecológica: tendências e perspectivas mundiais. In: KAGEYAMA, P. Y.; OLIVEIRA, R. E.; MORAES, L. F. D.; ENGEL, V. L.; GANDARA, F. B. Restauração ecológica de ecossistemas naturais. Botucatu: FEPAF, 2003. p. 1 - 26.

FERREIRA, D. F. SISVAR. Lavras: Departamento de Ciências Exatas, Universidade Federal de Lavras, 2004.

JACOMINE, P. K. T. Solos sob matas ciliares. In: RODRIGUES, R. R.; LEITÃO FILHO, H. F. (ed.). Matas ciliares: conservação e recuperação. 2.ed. São Paulo: Editora da Universidade de São Paulo, 2004. p. $27-31$.

JOLY, A. B. Conheça a vegetação brasileira. São Paulo: Polígono, 1970. v. 18, 181 p.

KAGEYAMA, P. Y.; DIAS, I. S. Aplicação da genética em espécies florestais nativas. Silvicultura em São Paulo, São Paulo, v. 16A, n. 2, p. 728 - 791, 1982.

LOPES, A. S. "Solos sob cerrados": características, propriedades e manejo. Piracicaba: Instituto da Potassa \& Fosfato: Instituto Internacional da Potassa, 1984. 162 p.

MARTINS, F. R. Fitossociologia de florestas do Brasil: um histórico bibliográfico. Pesquisa Série Botânica, São Leopoldo, v. 40, p. 104 - 164, 1989. 
PISSARA-FRANCO, T. C. T. Fenologia da embaúba (Cecropia pachystachya trec., Cecropiaceae) visando sua utilização na recomposição de matas ciliares. 1994. 53 f. (Trabalho de Graduação em Agronomia) - Faculdade de Engenharia de Ilha Solteira, Universidade Estadual Paulista, Ilha Solteira, 1994.

RAIJ, B. Van; QUAGGIO, J. A. Métodos de análises de solo para fins de fertilidade. Campinas: Instituto Agronômico, 1983. 31 p. (Boletim Técnico, 81).

SALVADOR, J. L. G. Considerações sobre as matas ciliares e a implantação de reflorestamentos mistos nas margens de rios e reservatórios. 2 ed. São Paulo: CESP/ARI, 1989. 15 p. (Série Divulgação e Informação, 105).

SCOTT, A. J.; KNOTT, M. A cluster analysis method for grouping means in the analysis of variance. Biometrics, v. 30, n. 3, p. 507 - 512, 1974.

SHEER, M. B. A decomposição e liberação de nutrientes da serapilheira foliar em um trecho da Floresta Ombrófila Densa Aluvial em regeneração em Guaraqueçaba (PR). Floresta, Curitiba, PR, v. 38, n. 2, p. 253 - 266, 2008.

SOUZA, Z. M. Propriedades físicas e químicas de um Latossolo Vermelho-Escuro de Selvíria (MS) sob diferentes usos e manejos. 2000. 127f. Dissertação (Mestrado) - Faculdade de Engenharia de Ilha Solteira, Universidade Estadual Paulista, Ilha Solteira, 2000.

TONIATO, M. T. Z.; LEITÃO FILHO, H. F.; RODRIGUES, R. R. Fitossociologia de um remanescente de floresta higrófila (mata de brejo) em Campinas, SP. Revista Brasileira de Botânica, São Paulo. v. 21, n. 2, 1998. 